\title{
Ação do fluoreto de dentifrícios sobre o esmalte dentário
}

\section{Action of the fluoride of dentifrices on the dental enamel}

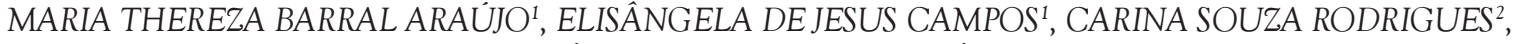 \\ LILIAN SALES SERRAVALLE², MAX JOSÉ P. LIMA3, DANILO B. ARAÚJO3.
}

${ }^{1}$ Professor de Bioquímica. Instituto de Ciências da Saúde. UFBA

${ }^{2}$ Bolsista de Iniciação Cientifica, Instituto de Ciências da Saúde, UFBA

${ }^{3}$ Acadêmico de Odontologia, Faculdade de Odontologia. UFBA

\section{Resumo}

A possibilidade de intervir na dissolução do esmalte dentário, diminuindo significativamente a perda de minerais, e de reverter o progresso de lesões cariosas iniciais torna o fluoreto um elemento indispensável à saúde dental. Enquanto recurso preventivo, o fluoreto é uma alternativa de saúde pública bucal extremamente eficaz e de baixo custo. Visando subsidiar a literatura que trata da eficácia do fluoreto de sódio $(\mathrm{NaF})$, do monofluorfosfato de sódio (MFP) e da associação entre o fluoreto de sódio e o monofluorfosfato de sódio (NaF/MFP) que integram os dentifrícios, o presente trabalho teve por objetivo determinar, in vitro, o grau de proteção do esmalte dentário humano, assegurado por estas formas de fluoreto, frente à queda do $\mathrm{pH}$. As taxas do cálcio liberado do esmalte previamente tratado, expressas em $\mathrm{mg} / \mathrm{dl} / 20 \mathrm{~min}$, revelaram o poder protetor destes fármacos. Os resultados experimentais comprovam que o fluoreto de sódio contido no dentifrício teste revelou maior proteção do esmalte, apesar da menor concentração deste fármaco em relação ao monofluorfosfato de sódio e à associação fluoreto de sódio/ monofluorfosfato de sódio, contidos nos demais. Tais conclusões sugerem a continuidade das experimentações, em face da importância de se obterem respostas eficazes, utilizando-se baixas concentrações da forma de fluoreto, comprovadamente mais efetiva, e de se realizar o controle de qualidade dos mais diversos produtos que são lançados no mercado freqüentemente.

\section{Palavras-chave}

Fluoreto, dentifricio, MFP, esmalte dentário, remineralização

Prof Maria Thereza Barral Araújo

Laboratório de Bioquimica Oral

Departamento de Biofunção

Instituto de Ciências da Saúde

Universidade Federal da Bahia

Av. Reitor Miguel Calmon s/n Vale do Canela

40.110-100 - Salvador, Bahia, Brasil

e-mail:tbarral@hotmail.com 


\section{Introdução}

Nos mais diversos países, inúmeros programas de promoção de saúde bucal utilizam o flúor como elemento de prevenção, em vista da capacidade anticárie desse elemento, associada à educação sanitária (ARAÚJO, 1995).

São indiscutiveis os benefícios do flúor para a preservação e a manutenção da saúde dos dentes, entretanto, em virtude das várias opções de utilização deste produto, deve-se estar atento às indicações, em função do risco de cárie inerente a cada individuo (KRASSE, 1988), como também aos cuidados para prevenir a fluorose (PALENSTEIN et al., 1997), em particular, em decorrência da excessiva ingestão de flúor pelas crianças (JOYSTON-BECHAL; KIDD, 1994).

É consensual, entre os pesquisadores, que o uso de dentifrícios fluoretados tem contribuido enormemente para o declínio da cárie dental nos países industrializados. A regularidade do uso dos dentifrícios assegura altas concentrações de fluoreto na interface placa-esmalte (BOWEN; TABAK, 1995; ELLWOOD; BLINKHORN; DAVIES, 1998). Existem extensas evidências de que a prevalência da cárie tem declinado, depois de passados vinte anos, na maioria dos paises industrializados (BOWEN; TABAK, 1995). Outrossim, a contribuição para a melhoria da saúde oral dada pelos dentifrícios fluoretados foi reconhecida recentemente pela Organização Mundial de Saúde.

Para que um dentifrício seja considerado eficiente para prevenção de cárie, ele precisa apresentar flúor disponivel, estável e reativo, de acordo com as especificações mínimas exigidas pela American Dental Association (ADA) (CURY, 1989). Normalmente, a concentração de flúor nos dentifrícios situa-se entre 1.000 e $1.500 \mathrm{ppmF}^{-}$, embora os resultados de determinados estudos clínicos, realizados com dentifrícios com baixo conteúdo de flúor (250 a 500 ppmF-), indiquem uma eficácia equivalente aos cremes dentais que contêm concentrações de flúor consensualmente consolidadas até então: 1.000 a 1.500 ppmF $^{-}$ (KOCH et al., 1982).
Estudo realizado, durante quatro semanas, com 16 crianças previamente selecionadas teve por objetivo avaliar o efeito do uso diário de dentifrício com baixa concentração de fluoreto - 250 ppm. A retenção de flúor, o desenvolvimento de lesões de cárie, assim como a prevalência de Lactobacillus e de S. mutans na placa dental acumulada em pré-molares homólogos foram analisados. Para tanto, foram adaptadas bandas ortodônticas, com o objetivo de favorecer a retenção de placa e o desenvolvimento de lesões de esmalte. Os dentes foram analisados posteriormente, através de espectômetro de massa iônica secundária, a fim de determinar o perfil da concentração de fluoreto e de outros elementos no esmalte e nas lesões. Quantidades significativas de flúor retido no esmalte foram detectadas; entretanto, o tamanho das lesões e a composição da placa não revelaram qualquer diferença em relação ao grupo controle tratado com dentifrício placebo. Apesar da retenção, pelo esmalte, do fluoreto contido no dentifrício, tal retenção não inibe significativamente o desenvolvimento de lesões de cárie, se uma placa dental cariogênica, com altos níveis de microrganismos produtores de ácido, permanece continuadamente aderida à superfície do esmalte (PETERSSON et al., 1995).

Observações clínicas, durante três anos, envolvendo 4.294 crianças com idades que variaram entre 11 e 12 anos no início das investigações, foram realizadas com o objetivo de se comparar a eficácia anticárie de dentifrícios contendo fluoreto de sódio, monofluorfosfato de sódio e a associação fluoreto de sódio/trimetafosfato de sódio, usando duas concentrações de fluoreto a 1.000 ppm de flúor e 1.500 ppm de flúor, respectivamente. Após três anos, foi constatado um aumento médio do incremento do Índice de superfícies cariadas, perdidas e obturadas (CPOS). O uso do dentifrício que continha apenas fluoreto de sódio resultou num indice $6,4 \%$ mais baixo quando comparado àquele decorrente do uso de dentifrício contendo monofluorfosfato de sódio. A diferença entre os individuos que usaram a combinação fluoreto de sódio/trimetafosfato de sódio e 
aqueles que utilizaram o monofluorfosfato de sódio foi de $8,1 \%$. Tais diferenças foram clínica e estatisticamente significativas e consistentes para os dois niveis de fluoreto testados. $O$ incremento médio do CPOS para os indivíduos que usaram a associação fluoreto de sódio e trimetafosfato de sódio foi $2 \%$ mais baixo em relação aos individuos que usaram apenas o fluoreto de sódio. Os exames radiográficos confirmaram as avaliações clínicas, ao demonstrarem a diferença de $8,1 \%$ entre os usuários do dentifrício que continha apenas o fluoreto de sódio e aqueles tratados pelo monofluorfosfato de sódio. A eficácia da associação do fluoreto de sódio com o trimetafosfato de sódio em comparação com a eficácia do monofluorfosfato de sódio revelou uma diferença da ordem de 2,3\%. Através deste estudo clínico, os autores concluíram que o fluoreto de sódio tem superioridade terapêutica, quando incorporado aos dentifrícios com base de sílica, em relação àqueles que contêm monofluorfosfato de sódio (STEPHEN et al., 1994).

Outro experimento clínico relevante, realizado durante três anos, na Flórida, comparou o efeito de quatro níveis de dose de monofluorfosfato de sódio e um único nivel de dose de fluoreto de sódio incorporados a dentifrícios, através dos indices CPOS, Índice de dentes cariados, perdidos e obturados (CPOD e Índice de superfícies cariadas e obturadas (COS) interproximais. 5.474 crianças participaram integralmente deste estudo, sendo submetidas a exames clinicos, exames radiológicos e escovações diárias supervisionadas nas escolas. Os resultados indicaram que o grupo tratado com $2.000 \mathrm{ppmF}^{-}$de fluoreto de sódio revelou um aumento do índice CPOS significativamente menor do que o grupo tratado com 2.000 $\mathrm{ppmF}^{-}$de monofluorfosfato de sódio, portanto uma superioridade significativa do fluoreto de sódio sobre o monofluorfosfato de sódio. Este estudo demonstrou também uma clara conexão entre o binômio dose-resposta e o nivel de fluoreto do monofluorfosfato de sódio contido nas formulações de dentifrícios com $1.000,1.500,2.000$ e $2.500 \mathrm{ppmF}^{-}$, respectivamente. A eficácia anticárie de dentifrícios con- tendo $2.000 \mathrm{ppmF}^{-} /$fluoreto de sódio, observada neste estudo, parece ser semelhante à eficácia assegurada por dentifrício contendo 2.500 $\mathrm{ppmF}^{-} /$monofluorfosfato de sódio. Portanto, a eficácia anticárie do dentifrício com monofluorfosfato de sódio aumenta com a elevação da concentração do fluoreto adicionado, enquanto a eficácia anticárie de um creme dental contendo $2.000 \mathrm{ppm}$ de flúor sob a forma de fluoreto de sódio é superior àquela do dentifrício com 2.000 ppm de flúor, na forma de monofluorfosfato de sódio (MARKS et al., 1994).

Vários estudos laboratoriais têm procurado relacionar a dureza do esmalte e o fluoreto incorporado a este tecido através de cremes dentais. Os efeitos de dentifrícios fluoretados sobre o desgaste de dentes pré-molares extraídos de humanos residentes em duas áreas geográficas com diferentes concentrações de flúor no suprimento da água de abastecimento foram criteriosamente avaliados. Nove dentes de cada área foram seccionados no sentido bucolingual, em pares combinados e submetidos a um regime de desgaste in vitro. A experimentação consistiu na imersão das secções dentárias, durante 5 minutos, numa solução de ácido cítrico a 6\%, tamponado em pH 3,5, seguida por 200 ciclos de escovação linear com um dentifrício fluoretado no grupo teste e com um dentifrício sem flúor no grupo controle. Todo este processo foi repetido 720 vezes. Os dentes coletados dos individuos residentes em Birmingham, onde o suprimento de flúor na água de abastecimento era de 1 ppm, revelaram uma concentração de fluoreto mais alta na superfície do esmalte, assim como maior dureza do que os dentes de individuos provenientes de Londres, onde a água de abastecimento não era fluoretada. As observações revelaram que o menor desgaste foi produzido naquelas estruturas dentárias tratadas pelo dentifrício fluoretado, quando comparadas às submetidas aos dentifrícios de formulação idêntica, contudo desprovidas de flúor ( $\mathrm{p}<0,001)$. Os resultados deste estudo levaram os pesquisadores a concluir que a intensidade do desgaste dentário in vitro não foi afetada em função de diferentes concentrações de 
flúor e da dureza do esmalte (BARTLETT; SMITH; WILSON, 1994).

Exames clinicos e radiológicos foram realizados em amostragem aleatória para 20\% de crianças com 12 anos, residentes em Reykjavik, Islândia, visando avaliar o efeito da escovação com dentifrício fluoretado, no periodo compreendido entre 1984 e 1991. Constatou-se ter havido um decréscimo na experiência de cárie da ordem de $60 \%$, confirmando a eficácia do fluoreto adicionado a dentifrícios (BJARNASON et al., 1993).

Com base nessas evidências, diversas investigações cientificas têm sido feitas, visando aumentar o poder de remineralização do esmalte através do ion fluoreto sob a forma de monofluorfosfato de sódio (MFP), contido nos dentifrícios. Dai porque, tendo em consideração a solubilidade dos sais de cálcio do monofluorfosfato de sódio, vêm-se desenvolvendo vários testes objetivando interferir diretamente no sistema abrasivo dos dentifricios, a fim de assegurar maior eficácia do monofluorfosfato de sódio (DE PAOLA, 1993). Outrossim, diversos ensaios realizados associando-se o MFP ao fosfato dicálcico diidratado (DCPD) resultaram num aumento dos niveis de fluoreto na saliva total e na placa, com conseqüente remineralização de lesões cariosas. Os resultados obtidos em laboratório, em animais e através de estudos in situ indicam que a combinação MFP/DCPD é a única formulação que promove uma supersaturação extra na saliva e na placa, aliada aos efeitos anticárie (GAFFAR; BLAKE-HASKINS; MELLBERG, 1993).

Trabalhando com amostras de esmalte de terceiros molares não erupcionados, submetidos à ação de soluções de dentifrícios contendo monofluorfosfato de sódio ou fluoreto de sódio durante 24 horas, detectou-se a formação de partículas semelhantes ao fluoreto de cálcio na superfície do esmalte, caracterizadas pela solubilidade em meio alcalino. Os experimentos mostraram que o material, parecido com o fluoreto de cálcio formado no esmalte, após o tratamento com o dentifrício contendo monofluorfosfato de sódio, provavelmente originou-se do fluoreto livre. Esta possibilidade de deposição moderada de fluoreto de cálcio tem promissor significado de natureza clinica, uma vez que os dentes são freqüentemente expostos a dentifrícios fluoretados. As experimentações realizadas não revelaram a ocorrência de fluoreto firmemente aderido ao esmalte tratado pelos cremes dentais contendo o monofluorfosfato de sódio (CRUZ; RÖLLA; ÖGAARD, 1994a). Portanto, em que pesem as investigações cientificas sinalizarem para um melhor desempenho do fluoreto de sódio comparado ao monofluorfosfato de sódio, a tendência atual na produção de dentifrícios é se considerar a concentração do fluoreto, a combinação de mais de um componente de flúor e a adição de agentes ativos que potencializem a ação deste ion (THYLSTRUP; FEJERSKOV, 1995). Para o aumento da substantividade dos fluoretos nos dentifrícios, freqüentemente têm-se associado estes produtos a grupos aminas que, em função das cargas positivas, ligam-se às cargas negativas disponiveis no dente e na película adquirida do esmalte, prolongando, desta maneira, a disponibilidade do flúor na superfície dentária. Esta substantividade pode ser reforçada em ambientação ácida, a fim de favorecer a deposição do flúor no esmalte: dai a introdução dos fluoretos acidulados (LOESCHE, 1993).

Ensaios laboratoriais vêm sendo feitos visando à obtenção de dentifrícios contendo associações do fluoreto a anti-sépticos e a produtos antiplaca, tais como o cloreto de cetilpiridinio (NEWBRUN, 1991; PINHEIRO, 1991), o triclosan/gantrez (TEN CATE, 1993; WALKER, 1994; HAWLEY et al., 1995) e a clorexidina (JOYSTON-BECHAL et al., 1992; YATES et al., 1993; BOWEN; TABAK, 1995). Experimentos realizados, visando a comparar os efeitos de um dentifricio controle não fluoretado (negativo) com os de um dentifricio controle fluoretado (positivo) contendo $1.000 \mathrm{ppm}$ de flúor sob a forma de monofluorfosfato de sódio e com um dentifrício teste contendo este mesmo teor de flúor associado ao triclosan a $0,3 \%$ e ao citrato de zinco trihidratado (ZCT) a $0,75 \%$, avaliaram o desempenho farmacológico destes produtos. Foram realizados os testes de viabilidade biológica do fluoreto e de lesão de 
esmalte, assim como estudos de remineralização (ciclo do $\mathrm{pH}$ ) e de cárie in situ. Os resultados mostraram que os dois dentifrícios fluoretados tiveram melhor desempenho em todos os testes, em relação ao controle negativo. Não foram observadas diferenças entre os dois cremes dentais fluoretados nos estudos in vivo. Sob as condições de desafio cariogênico, a progressão da cárie in situ foi inibida pelo dentifrício teste contendo fluoreto/triclosan/ZCT, enquanto foi observado um aumento da severidade da lesão no grupo tratado com o dentifrício que continha apenas o flúor (TEN CATE, 1993).

Do mesmo modo, um estudo que avaliou os efeitos de um dentifrício contendo $0,3 \%$ de triclosan, 2\% de gantrez e 0,243\% de fluoreto de sódio sobre a composição microbiana da placa supragengival de humanos concluiu ter havido altas reduções estatisticamente significativas da microflora desta placa, embora não tivesse sido detectada uma diminuição significativa na contagem total dos microrganismos anaeróbios e daqueles estritamente anaeróbios. Não houve alterações na composição microbiana da flora normal, nem o aparecimento de patógenos periodontais ou oportunistas (WALKER, 1994). Outros estudos importantes vêm sendo realizados, com a finalidade de confirmar a eficácia do dentifrício com $0,24 \%$ de fluoreto de sódio e 0,3\% de triclosan/gantrez (HAWLEY et al., 1995).

Assim é que, sob a forma de fluoreto de sódio, de monofluorfosfato de sódio ou da associação de fluoreto de sódio e de monofluorfosfato de sódio, os dentifrícios devem apresentar uma concentração de flúor que varie de $525 \mathrm{ppmF}^{-}$a $1.500 \mathrm{ppmF}^{-}$. Considerando-se a confiabilidade dos produtos comerciais, a preocupação deve dirigir-se à indicação, a fim de reduzir o risco de fluorose. A ingestão de dentifrícios fluoretados por crianças jovens, pré-escolares, deve ser, seguramente, o fator que mais contribui para a fluorose dental nos Estados Unidos (LEVY; MAURICE; JAKOBSEN, 1993).

Sob supervisão, crianças muito jovens (menos de 5 anos) devem utilizar dentifrícios com 500 a 600 ppm de flúor. As crianças acima de 8 anos, por estarem menos vulneráveis à fluorose dentária, podem utilizar-se dos dentifrícios de natureza familiar contendo 1.000 a $1.450 \mathrm{ppm}$ de flúor, salvo se estão em uso de suplementos fluoretados; por integrarem grupos de pacientes de alto risco de cárie é que se recomenda o uso de dentifrícios com baixo teor de flúor e relativa supervisão (JOYSTON. BECHAL; KIDD, 1994). Tal preocupação está registrada em recentes publicações que relatam a ocorrência de fluorose dental em crianças préescolares consideradas de alto risco e que ingeriram dentifrício fluoretado (LEVY, 1993). Estudos clínicos e radiológicos, realizados em 350 crianças, com idade média de 7 anos e meio, em Perth, Austrália, avaliaram a ocorrência de cárie e de fluorose, uma vez que essas crianças haviam recebido flúor, no período compreendido entre o nascimento e os 4 anos de idade, através da água de abastecimento e com o uso mínimo de suplementos fluoretados. A análise dos resultados revelou a ação protetora do íon fluoreto, ao tempo em que a utilização da água fluoretada de abastecimento não foi considerada um significativo fator de risco de fluorose. Todavia, a interrupção da amamentação aos 9 meses de idade e o prazer de deglutir dentifrício foram fatores de risco considerados estatisticamente significativos (RIORDAN, 1993).

Em face desses dados, é de significativa importância a continuidade das investigações sobre a eficácia das formas químicas pelas quais o fluoreto é incorporado aos dentifrícios, enxaguatórios, géis, vernizes e demais veículos. Seguramente, investigações cientificas desta natureza contribuirão para maiores esclarecimentos a respeito da eficácia do fluoreto de sódio $(\mathrm{NaF})$, comparativamente àquela assegurada pelo monofluorfosfato de sódio (MFP). A importância desse tema assume maior relevância a partir do momento em que os dentifrícios e os enxaguatórios comercializados encerram, em suas formulações, o fluoreto de sódio, o monofluorfosfato de sódio ou, ainda, a associação de ambos. Freqüentemente, o mesmo agente fluoreto está presente em diferentes concentrações em diversos produtos de uso doméstico, necessitando, portanto, avaliações cada vez mais acuradas, com vistas a que se oriente a prescrição de produtos cuja eficácia das doses, se 
possivel mínimas, atendam às exigências preventivas e/ou terapêuticas. Há que se considerar, também, a importância da realização de investigações cientificas como o único recurso de reconhecida confiabilidade para o controle da qualidade desses produtos, entre as quais aquelas realizadas in vitro, o que justifica o presente estudo.

\section{Materiais e métodos}

O presente trabalho avaliou o grau de proteção do esmalte dentário humano em pó pelo ín fluoreto contido nos quatro dentifrícios teste Kolynos Ação Total (DI), Kolynos Super Branco (DII), Kolynos Flúor 2 Gel (DIII) e Colgate com $\mathrm{Na}_{2} \mathrm{CO}_{3}$ (DIV), comparados à ação dos dentifrícios controle, sem fluoreto, Phillips (CI) e Sensodyne (CII). Esses dentifrícios contêm fluoreto nas seguintes formas: DI $=1.100 \mathrm{ppmF}^{-} \mathrm{NaF}$; DII $=1.200$ $\mathrm{ppmF}^{-} \mathrm{MPF}$; DIII $=1.500 \mathrm{ppmF}^{-} \mathrm{NaF} / \mathrm{MPF}$ e $\mathrm{DIV}=1.450 \mathrm{ppmF}^{-} \mathrm{MPF}$. O dentifricio controle CI possui o $\mathrm{pH}$ alcalino 10,10 , enquanto o dentifrício CII possui o $\mathrm{pH} 7,02$. Amostras de esmalte tratadas por cada dentifrício foram submetidas, individualmente, à ação de pH 6,8, 6,0, 5,0 e 4,0, tendo as taxas do cálcio liberado, determinadas colorimetricamente, traduzido o grau de proteção do esmalte dentário pelo fluoreto. A escolha desses valores de $\mathrm{pH}$ decorreu da proposição de se observar o grau de proteção do esmalte pelo íon fluoreto submetido ao $\mathrm{pH}$ próximo à neutralidade (6.8) frente à queda deste fator aos níveis considerados significativamente baixos.

\section{Extração e isolamento do esmalte}

Visando extrair e isolar o esmalte dentário humano, a técnica de separação dos tecidos dentários, fundamentada nas diferentes densidades desses tecidos (TASTALDI apud ARAÚJO, 1995, p. 78), foi adaptada para a realização do presente estudo.

Para a execução dos ensaios experimentais, foram selecionados dentes humanos permanentes, recém-extraidos. Rigorosamente limpos e sem impurezas, os dentes foram secos em estufa a $37^{\circ} \mathrm{C}$, durante 24 horas, sendo, em seguida, triturados no aparelho Chaterbox, equipamento de origem francesa, e tamisados em tamis de malha cem.
O pó dentário fino e uniforme, obtido com sucessivas triturações e tamisações, foi tratado pela mistura bromofórmio/acetona, e centrifugado sucessivas vezes a $3.000 \mathrm{rpm}$ (Centrifugador Fanen, Excelsa Baby I, modelo 206), visando a separação e o isolamento do esmalte.

\section{Protocolo experimental}

A técnica da influência do íon fluoreto na solubilidade do esmalte dentário submetido ao meio ácido foi adaptada, visando a avaliação da ação protetora dos seis dentifrícios, sendo quatro deles fluoretados (DI, DII, DIII e DIV), numa amostra de dez experimentos.

O esmalte dentário humano em pó foi submetido à ação dos seis dentifrícios sob a forma de soluções, na proporção de uma parte de cada produto para três partes de água desionizada. Com o objetivo de se estudar a ação do íon fluoreto sobre esmalte dentário em pó, esta estrutura foi tratada por cada dentifrício, durante 20 minutos, sob forte agitação mecânica, automática (homogeneizador modelo Homolab, fabricado pela BIO ENG Indústria e Comércio), seguindo-se sucessivas centrifugações a 3.000 rpm e ressuspensão dos sedimentos em água desionizada.

Aos sedimentos finais obtidos, foi adicionado o tampão acetato $0,2 \mathrm{M}$ para o ajuste dos valores de $\mathrm{pH}$ necessários à realização dos testes

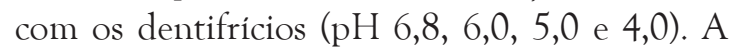
ação dos diferentes níveis de $\mathrm{pH}$ sobre os respectivos sedimentos se desenvolveu durante 20 minutos, sob forte agitação mecânica, automática (Homolab).

A avaliação da ação protetora do íon fluoreto contido nos dentifrícios testados foi realizada mediante a exposição do esmalte dentário humano, previamente influenciado por esses produtos, frente à descalcificação imposta com a queda do $\mathrm{pH}(6,8,6,0,5,0$ e 4,0). A escolha recaiu sobre o $\mathrm{pH} 4,0$ porque, sendo extremamente inferior ao nivel considerado crítico, representa um desafio cariogênico extremamente drástico (KRASSE, 1988; THYLSTRUP; FEJERSKOV, 1995). 
Após a centrifugação a $3.000 \mathrm{rpm}$, durante 10 minutos, os sedimentos de esmalte foram descartados e, em alíquotas dos sobrenadantes coletados, foram determinadas as taxas do cálcio total liberado do esmalte dentário humano devidamente tratado pelos dentifrícios e submetido aos diferentes valores de $\mathrm{pH}$, a fim de avaliar o grau de desmineralização dessa estrutura dentária.

\section{Análises bioquímicas}

O método colorimétrico escolhido para a realização das dosagens do cálcio total, denominado método da orto-cresolftaleína complexonona, fundamenta-se na formação do complexo corado entre o cálcio e a o-cresolftaleína em $\mathrm{pH}$ alcalino. Foram utilizados kits comerciais Doles, fabricados pela Doles Reagente, Goiânia-Goiás, e Bioclin, fabricado pela Quibasa - Química Básica Ltda., Belo Horizonte-Minas Gerais, para realizar as dosagens do cálcio liberado do esmalte tratado pelos dentifrícios. Tais escolhas decorreram da procedência industrial e do controle de qualidade desses produtos, uma vez que os mesmos são estandartizados. As taxas do cálcio total, presente nas alíquotas dos sobrenadantes coletados, são expressas em miligramas por decilitro, em função do tempo de 20 minutos ( $\mathrm{mg} \mathrm{Ca}^{2+} / \mathrm{dl} / 20 \mathrm{~min}$ ). Para cada experimento, as dosagens de cálcio foram realizadas em triplicata e lidas em espectrofotômetro Micronal, modelo B382.

\section{Análises estatísticas}

Os resultados são apresentados como médias e erros padrão da média (EPM). As médias dos diversos grupos foram comparadas por análise de variância (ANOVA) de medidas repetidas, levando-se em consideração a comparação entre o pH (fator A) e a concentração de cálcio (fator B), seguida do teste paramétrico de Scheffé. Considerou-se, como diferença estatisticamente significativa, um valor de $\mathrm{p}<0,01$, sendo $\mathrm{N}=10$.

\section{Resultados}

A avaliação dos dados revela a eficácia dos dentifrícios fluoretados testados DI, DII, DIII e DIV em comparação com os dentifrícios CI e CII, que não possuem o fluoreto em suas formulações.

Outrossim, para a análise dos dados, estão sendo considerados como referenciais o dentifrício teste DI e o dentifrício teste DIII, em razão do melhor desempenho desses produtos, com destaque para o dentifrício DI, uma vez que a proteção assegurada ao esmalte pelo flúor neles contido resultou na liberação das mais baixas taxas de cálcio frente à queda do $\mathrm{pH}$.

No tocante à proteção do esmalte dentário humano, esta proteção está representada através de taxas médias e EPM das dosagens do cálcio total (mg $\mathrm{Ca}^{2+} / \mathrm{dl} / 20 \mathrm{~min}$ ) liberado do esmalte, previamente influenciado pelos cremes dentais e submetido à ação de diferentes níveis de $\mathrm{pH}(6,8,6,0,5,0$ e 4,0).

As TAB. 1 e 2 expressam os dados referentes à influência dos dentifrícios controle CI e CII - sobre o esmalte dentário submetido à ação dos valores decrescentes de $\mathrm{pH}(\mathrm{p}<$ 0,01).

TABELA 1

Taxas médias e EPM das dosagens do cálcio total ( $\mathrm{mg} / \mathrm{dl} / 20 \mathrm{~min})$ liberado do esmalte dentário humano tratado pelos dentifricios CI e CII (controle) e DI, DII, DIII, DIV (teste), frente à ação de pH 6,8, 6,0, 5,0 e 4,0

\begin{tabular}{|c|c|c|c|c|}
\hline DENTIFRÍCIO & $\mathrm{pH}$ & 6,0 & 5,0 & 4,0 \\
\hline $\mathrm{CI}$ & $16,92 \pm 5,41$ & $33,82 \pm 1,35$ & $37,81 \pm 1,27$ & $37,62 \pm 1,50$ \\
\hline CII & $24,95+0,77$ & $29,16 \pm 1,34$ & $35,60 \pm 0,65$ & $36,20+1,10$ \\
\hline DI & $2,64 \pm 0,85$ & $4,05 \pm 1,59$ & $7,50 \pm 0,70$ & $11,81 \pm 1,32$ \\
\hline DII & $15,03 \pm 0,80$ & $39,64 \pm 2,50$ & $42,24 \pm 2,14$ & $42,08 \pm 2,20$ \\
\hline DIII & $5,85 \pm 1,61$ & $8,94+0,81$ & $21,38+1,60$ & $31,71+2,85$ \\
\hline DIV & $9,58 \pm 1,31$ & $31,71 \pm 2,84$ & $35,21 \pm 3,16$ & $35,69 \pm 2,41$ \\
\hline
\end{tabular}


TABELA 2

Análise de cada grupo frente à variação do $\mathrm{pH}$

\begin{tabular}{|c|c|c|c|c|c|c|c|}
\hline $\mathrm{pH}$ & GRUPO & CI & CII & D I & D II & D III & D IV \\
\hline $6.8 \times 6.0$ & & s & ns & ns & $\mathrm{s}$ & ns & s \\
\hline $6.8 \times 5.0$ & & $\mathrm{~s}$ & ns & ns & s & s & s \\
\hline $6.8 \times 4.0$ & & $\mathrm{~s}$ & $\mathrm{~ns}$ & $\mathrm{~ns}$ & $\mathrm{~s}$ & $\mathrm{~s}$ & $\mathrm{~s}$ \\
\hline
\end{tabular}

As médias dos diversos grupos foram comparadas por análise de variância (ANOVA) de medidas repetidas, levando-se em consideração a comparação entre o $\mathrm{pH}$ (fator $\mathrm{A}$ ) e a concentração de cálcio (fator B). Fator $\mathrm{A}: \mathrm{F}=18,82877 ; \mathrm{p}<0,0001$. Fator B; $F=52,11,666 ; p<0,0001$. Fator $A \times$ Fator $B: F=80.41741 ; p<0,0001$. Em seguida, tratando-se de múltiplas comparações, procedeu-se o teste paramétrico de Scheffé. s indica a diferença estatisticamente significativa e nַs indica que a diferença não é considerada estatisticamente significativa entre as taxas do cálcio liberado do esmalte tratado pelos dentifricios teste e controle, nos niveis de pH 6.0, 5.0 e 4.0, em comparação com as taxas do cálcio liberado em pH $6.8(\mathrm{p}<0,01) . \mathrm{N}=10$.

As taxas de cálcio referentes à influência exercida pelo dentifrício controle CI sobre o esmalte dentário humano submetido à ação de $\mathrm{pH}$ 6,0, 5,0 e 4,0 revelaram diferenças estatisticamente significativas em relação às taxas que decorreram da influência do pH 6,8 (p < $0,01)$, enquanto as taxas de cálcio referentes ao dentifrício controle CII não revelaram diferenças estatisticamente significativas, uma vez comparados os valores obtidos em $\mathrm{pH}$ 6,0, 5,0 e 4,0, em relação ao pH 6,8, conforme se explicita nas TAB. 1 e 2.
Outrossim, as taxas de cálcio que resultaram do esmalte dentário humano influenciado pelos dentifrícios controle não fluoretados CI e CII, quando comparadas entre si, não revelaram diferenças estatisticamente significativas, independentemente da queda do $\mathrm{pH}(6,8,6,0$, 5,0 e 4,0) - TAB. 1 e 3.

A análise destes resultados demonstra que os dentifrícios controle (CI e CII), do ponto de vista estatístico, apresentaram a mesma influência sobre o esmalte dentário em cada um dos quatro valores de $\mathrm{pH}$.

TABELA 3

Comparação entre os grupos em cada nivel de $\mathrm{pH}$

\begin{tabular}{|c|c|c|c|c|}
\hline GRUPO & 6,8 & 6,0 & 5,0 & 4,0 \\
\hline CI x CII & ns & ns & ns & ns \\
\hline CI x DI & $\mathrm{s}$ & $\mathrm{s}$ & $\mathrm{s}$ & $\mathrm{s}$ \\
\hline CI x DII & ns & ns & ns & ns \\
\hline CI $x$ DIII & ns & $\mathrm{s}$ & $\mathrm{s}$ & ns \\
\hline CI x DIV & ns & ns & ns & ns \\
\hline CII x DI & $\mathrm{s}$ & $\mathrm{s}$ & $\mathrm{s}$ & $\mathrm{s}$ \\
\hline CII x DII & ns & $\mathrm{ns}$ & ns & ns \\
\hline CII x DIII & $\mathrm{s}$ & $\mathrm{s}$ & $\mathrm{s}$ & ns \\
\hline CII $x$ DIV & $\mathrm{s}$ & ns & ns & ns \\
\hline DI x DII & ns & $\mathrm{s}$ & $\mathrm{s}$ & $\mathrm{s}$ \\
\hline DI $x$ DIII & ns & ns & ns & s \\
\hline DI x DIV & $\mathrm{ns}$ & $\mathrm{s}$ & $\mathrm{s}$ & $\mathrm{s}$ \\
\hline DII $x$ DIII & ns & s & s & ns \\
\hline DII x DIV & ns & $\mathrm{s}$ & ns & ns \\
\hline DIII x DIV & ns & $\mathrm{s}$ & $\mathrm{ns}$ & ns \\
\hline
\end{tabular}

As médias dos diversos grupos foram comparadas por análise de variância (ANOVA) de medidas repetidas, levando-se em consideração a comparação entre o pH (fator A) e a concentração de cálcio (fator B). Fator A: $F=18,82877$; p < 0,0001. Fator B; $\mathrm{F}=52,11,666 ; \mathrm{p}<0,0001$. Fator $\mathrm{A} \times$ Fator $\mathrm{B}: \mathrm{F}=80.41741 ; \mathrm{p}<0,0001$. Em seguida, tratando-se de múltiplas comparações, procedeu-se o teste paramétrico de Scheffé. $\underline{s}$ indica a diferença estatisticamente significativa e ñ indica que a diferença não é considerada estatisticamente significativa entre as taxas do cálcio liberado do esmalte tratado pelos dentifricios teste e controle, nos niveis de $\mathrm{pH} 6.8,6.0,5.0$ e $4.0(\mathrm{p}<0,01) . \mathrm{N}=10$. 
Quanto ao fato de as taxas de cálcio obtidas do esmalte tratado pelo dentifrício controle CII (sem fluoreto) não terem revelado diferenças estatisticamente significativas em qualquer dos quatro níveis de $\mathrm{pH}$ testados, há que se levar em consideração que, entre estes dois produtos (CI e CII), o dentifricio controle CII foi o que demonstrou a menor influência sobre o esmalte dentário humano, até mesmo frente a $\mathrm{pH} 6,8$.

As TAB. 1 e 3 referem-se à análise comparativa da influência dos quatro dentifrícios teste sobre o esmalte dentário humano submetido aos valores decrescentes de $\mathrm{pH}$ 6,8, 6,0, 5,0 e 4,0, em relação aos dentifrícios controle CI $(\mathrm{p}<0,01)$ e CII $(\mathrm{p}<0,01)$.

Conforme os dados das TAB. 1 e 3, em $\mathrm{pH} 6,8$, o dentifrício DI foi o único que revelou diferença estatisticamente significativa em relação ao dentifrício $\mathrm{CI}$, em face dos baixos valores do cálcio liberado do esmalte influenciado por aquele creme dental teste $(\mathrm{p}<0,01)$.

Em pH 6,0, os dentifrícios fluoretados DI e DIII foram os produtos que revelaram maior influência sobre o esmalte dentário, uma vez que as baixas taxas do cálcio liberado apresentaram diferenças estatisticamente significativas, em relação ao dentifrício controle CI ( $\mathrm{p}<0,01)$; os crescentes valores do cálcio liberado do esmalte influenciado pelos dentifrícios teste DII e DIV não revelaram diferença significativa, uma vez comparados ao dentifrício controle CI (TAB. 1 e 3$)$.

A avaliação das taxas do cálcio liberado em pH 5,0 revelou o mesmo fenômeno ocorrido em pH 6,0, conforme demonstram os dados das TAB. 1 e 3.

Em pH 4,0, apenas o dentifrício teste DI ainda demonstrou influenciar positivamente o esmalte, uma vez que os valores inferiores de cálcio decorrentes da influência deste produto fluoretado sobre este tecido indicaram diferenças estatisticamente significativas, em relação ao dentifrício controle CI ( $<<0,01)$ (TAB. 1 e 3).

Em pH 6,8, os dentifrícios fluoretados DI, DIII e DIV revelaram diferenças estatisticamente significativas quando comparados ao denti- frício controle CII ( $p<0,01)$, em face das baixas taxas de descalcificação do esmalte influenciado por esses dentifrícios teste. Em pH 6,0, os dentifrícios fluoretados DI e DIII foram os que revelaram maior proteção do esmalte dentário, uma vez que as baixas taxas do cálcio liberado apresentaram diferenças estatisticamente significativas, em relação ao dentifrício controle CII $(\mathrm{p}<0,01)($ TAB. 1 e 3).

O aumento das taxas do cálcio liberado do esmalte tratado pelos dentifrícios teste DII e DIV não revelaram diferenças significativas do ponto de vista estatístico, quando comparadas àquelas liberadas do esmalte tratado pelo dentifrício controle CII em pH 6,0, conforme está demonstrado nas TAB. 1 e 3. A influência positiva produzida pelos dentifrícios teste DI e DIII em pH 6,0 repetiu-se em pH 5,0 (p < 0,01). $O$ dentifrício teste DI foi o produto que revelou o maior poder de proteção do esmalte dentário, quando se submeteu este tecido, previamente tratado pelo dentifrício, ao $\mathrm{pH}$ 4,0. Os baixos valores de cálcio que foram detectados revelaram diferenças estatisticamente significativas em relação às taxas do cálcio liberado do esmalte tratado pelo dentifrício controle C II $(\mathrm{p}<0,01)$.

Ainda de acordo com as TAB. 1 e 3, a menor liberação de cálcio em pH 6,8 decorreu do esmalte influenciado pelos dentifrícios fluoretados DI e DIII. O esmalte tratado pelo dentifrício fluoretado DI, ao ser submetido ao $\mathrm{pH} 6,8$, liberou os menores valores de cálcio em relação aos demais dentifrícios teste. A influência decorrente da ação do dentifrício fluoretado DI sobre o esmalte dentário humano, em comparação com os demais produtos fluoretados (DII, DIII e DIV), não revelou diferenças de descalcificação consideradas estatisticamente significativas. Em relação aos cremes dentais fluoretados DII e DIV, os índices do cálcio liberado do esmalte tratado pelo dentifrício fluoretado DIII não revelaram diferenças estatisticamente significativas. De acordo com estes resultados, e do ponto de vista estatístico, os dentifrícios DI, DII, DIII e DIV, comparados entre si, revelaram a mesma influência sobre o esmalte dentário humano em $\mathrm{pH}$ 6,8. 
Em pH 6,0, o esmalte previamente tratado pelo dentifrício teste DI liberou as mais baixas taxas de cálcio. Quando comparadas estas taxas com as baixas taxas do cálcio liberado do esmalte influenciado pelo dentifrício teste DIII, a diferença não foi considerada estatisticamente significativa. A influência do esmalte pelos dentifricios fluoretados DI e DIII em relação aos dentifrícios fluoretados DII e DIV é evidente, uma vez que as diferenças revelaram-se estatisticamente significativas $(p<0,01)$. Estes dados confirmam a maior influência dos dentifrícios fluoretados DI e DIII sobre o esmalte, em relação aos demais cremes dentais em pH 6,0 (TAB. 3).

Conforme os dados das TAB. 1 e 3, em $\mathrm{pH} 5,0$, o esmalte previamente tratado pelo dentifrício fluoretado DI liberou as mais baixas taxas de cálcio, em comparação com as liberadas do esmalte dentário tratado pelo dentifricio fluoretado DIII. Contudo essa diferença não foi considerada estatisticamente significativa.

Neste mesmo pH, o dentifrício DI revelou superioridade de influência sobre o esmalte em relação aos dentifrícios DII e DIV ( $p<0,01)$, enquanto o dentifrício DIII exerceu maior influência apenas quando comparado ao produto DII. Essa diferença foi estatisticamente significativa, de acordo com o valor de $\mathrm{p}<0,01$. Muito embora o dentifrício DIII tenha revelado uma maior influência sobre o esmalte em relação ao dentifrício DIV, essa diferença não foi considerada significativa.

Os dados das TAB. 1 e 3 apontam o dentifrício fluoretado DI como o único que revelou influir, efetivamente, sobre o esmalte dentário humano, mesmo com a queda do $\mathrm{pH}$ para 4.0, de acordo com as baixas taxas do cálcio liberado, em comparação com todos os demais dentifrícios fluoretados. A diferença decorrente da influência exercida pelo dentifrício fluoretado DI sobre o esmalte dentário, em relação aos outros três cremes dentais testados (DII, DIII e DIV), foi significativa ( $<<0,01)$. Em pH 4,0 a influência exercida pelo dentifrício teste DIII em comparação com a influência dos dentifrícios teste DII e DIV não revelou diferenças estatisticamente significativas.
A confirmação da maior influência do dentifrício teste DI sobre os demais é também constatada através das baixas taxas do cálcio liberado do esmalte tratado por esse produto após ser submetido aos níveis de pH 6,8, 6,0, 5,0 e 4,0. Embora estas taxas tenham sido diferentes entre si e relativamente progressivas com a queda do $\mathrm{pH}$, tais diferenças não são significativas, como pode ser constatado nos dados das TAB. 1 e 2 .

Em que pese o dentifrício fluoretado DIII ter revelado níveis de influência próximos àqueles demonstrados pelo dentifrício fluoretado DI, pelo menos em pH 6,8 e 6,0, e superiores àqueles resultantes da ação dos demais dentifrícios teste (DII e DIV), este produto revelou igual influência sobre o esmalte dentário perante a faixa de $\mathrm{pH}$ 6,8 a 6,0, conforme demonstra a TAB. 3. Outrossim, as taxas do cálcio liberado do esmalte tratado pelo dentifrício teste DIII como conseqüência da queda do $\mathrm{pH}$ para os níveis 4,0 e 5,0 revelaram diferenças estatisticamente significativas, quando comparadas às taxas decorrentes da descalcificação ocorrida em pH 6,8 (p<0,01), ainda de acordo com as TAB. 1 e 2 .

Em relação aos dentifrícios fluoretados DII e DIV, o aumento da liberação das taxas de cálcio foi claramente evidenciado a cada queda do $\mathrm{pH}$. Logo, as diferenças entre os valores do cálcio detectados após a ação do pH 6,0, 5,0 e 4,0 foram significativas quando comparados esses valores aos do cálcio liberado em pH 6,8 (TAB. 1 e 2).

\section{Discussão}

Os resultados do presente estudo revelaram a eficácia do íon fluoreto em induzir proteção ao esmalte dentário humano submetido à ação de valores decrescentes de $\mathrm{pH}$, na faixa de 6,8 a 4,0. O estudo envolvendo os quatro dentifrícios fluoretados, amplamente comercializados no Brasil, particularmente em Salvador, Bahia, demonstraram diferentes eficácias entre esses veículos de aplicação tópica.

Outrossim, a análise da formulação dos dentifrícios utilizados nesse trabalho revelou não apenas a presença do flúor na forma de fluoreto 
de sódio, de monofluorfosfato de sódio ou da associação entre o fluoreto de sódio e o monofluorfosfato de sódio, como também a ocorrência de cada uma dessas formas em diferentes concentrações.

A possibilidade de interferir na dissolução do esmalte, diminuindo significativamente a perda de minerais e de reverter lesões cariosas iniciais, torna o ín fluoreto um elemento importante para a preservação da saúde dental, tendo em vista esta propriedade de natureza anticariogênica. Sob este aspecto, os resultados que integram o presente estudo, ao demonstrarem a capacidade de proteção do esmalte pelo fluoreto, estão de acordo com as diversas observações e experimentações clínicas e laboratoriais, conforme relatos de vários autores, entre os quais os de De Paola (1993), Kingman (1993), Cruz; Rölla e Ögaard (1994b); Luoma et al. (1994); Shellis e Duckworth (1994); Ten Cate e Marsh (1994); Bowen e Tabak (1995); Christofferson et al. (1995) e Cutress et al. (1995).

A ação protetora do íon fluoreto contido nos dentifrícios, demonstrada in vitro mediante o decréscimo das taxas do cálcio total liberado do esmalte dentário humano submetido ao meio ácido, deveu-se ao princípio que considera que a presença do fluoreto nas soluções ácidas subsaturantes de cálcio e de fosfato diminui a solubilidade do esmalte, pois, mesmo em pH ácido, a presença deste elemento compensará, parcialmente, a perda de cálcio e de fosfato na forma de hidroxiapatita, pelo ganho de cálcio, fosfato e fluoreto, em virtude da manutenção do meio supersaturado em relação ao produto de solubilidade da fluorhidroxiapatita (CURY, 1989). Os resultados desta ação protetora, baseada na metodologia experimental in vitro utilizada, estão de acordo com diversos relatos, entre os quais os de Serra et al. (1989); Imfeld et al. (1993); Kuroiwa et al. (1993); Kambara e Norde (1995); Chadwich e Gordon (1995); Fischer et al. (1995) e Wiltshire e Rensburg (1995).

Segundo Bowen e Tabak (1995), estudos realizados in vitro, in situ e em animais de laboratório revelaram consistentes resultados clini- cos favoráveis ao fluoreto de sódio $(\mathrm{NaF})$ em relação ao monofluorfosfato de sódio (MFP), em que pesem alguns ensaios clinicos com dentifrícios contendo o fluoreto de sódio ou o monofluorfosfato de sódio terem demonstrado substanciais reduções de lesões incipientes de esmalte, muito próximas. Estudos realizados em animais, comparando os efeitos de dentifrícios contendo fluoreto de sódio com cremes dentais que encerram monofluorfosfato de sódio, revelaram que os primeiros são invariavelmente superiores àqueles com monofluorfosfato de sódio. De acordo com esse autor, experimentações usando modelos intra-orais em humanos têm demonstrado que os dentifrícios com fluoreto de sódio resultam em deposições de fluoreto de cálcio significativamente maiores, do que com o monofluorfosfato de sódio, em lesões incipientes de esmalte. Ainda segundo Bowen e Tabak (1995), meta-análises de estudos clínicos comparando o fluoreto de sódio diretamente com o monofluorfosfato de sódio têm demonstrado que o fluoreto de sódio é mais eficaz do que o monofluorfosfato de sódio, em cerca de $6,8 \%$ a $7 \%$.

Particularmente, com relação à ação dos dentifrícios avaliados nesse estudo realizado in vitro, constata-se maior proteção do esmalte dentário pelo fluoreto de sódio em comparação com o monofluorfosfato de sódio.

Analisados, nas TAB. 1 e 2, os valores do cálcio liberado do esmalte influenciado pelo dentifrício não fluoretado CI, constata-se que as taxas do cálcio determinado após a ação do pH 6,0, 5,0 e 4,0 comparadas ao liberado em $\mathrm{pH} 6,8$, apresentam diferenças estatisticamente significativas $(\mathrm{p}<0,01)$. Contudo, esse fenômeno não se repetiu com a aplicação do dentifrício CII, uma vez que as taxas do cálcio resultantes da ação de pH 6,0, 5,0 e 4,0 não diferiram significativamente, do ponto de vista estatístico, em relação aos valores do cálcio liberado em $\mathrm{pH} 6,8$.

Em que pese não ter significância estatística a diferença entre os valores do cálcio liberado do esmalte tratado pelos dentifrícios controle CI e CII em pH $6.8(16,92+5,41$ e 
$24,95 \pm 0,77$, respectivamente), possivelmente as taxas do cálcio liberado do esmalte tratado pelo dentifrício controle CI foram menores, em virtude do tamponamento assegurado por este produto, uma vez que o mesmo detém um $\mathrm{pH}$ extremamente alcalino (10.10), enquanto o dentifrício controle CII possui o $\mathrm{pH}$ praticamente neutro (7.02). Dessa forma, provavelmente, o dentifrício controle CI contribuiu efetivamente para o tamponamento, reduzindo o grau de descalcificação do esmalte dentário submetido ao pH 6.8. Outrossim, é possível que esta ação protetora tenha deixado de ocorrer com a queda do $\mathrm{pH}$ a que se submeteu o esmalte tratado, conforme está expresso na TAB. 1 .

De acordo com a TAB. 3, em pH 6,8, os graus de proteção do esmalte dentário tratado pelos dentifrícios fluoretados DI, DII, DIII e DIV não revelaram diferenças estatisticamente significativas. Este fato decorreu, provavelmente, não só da presença do fluoreto nessas formulações, mas, principalmente, em virtude do $\mathrm{pH}$ do meio ser próximo à neutralidade. Esse fenômeno independeu da forma pela qual o flúor tenha estado presente - fluoreto de sódio, monofluorfosfato de sódio ou fluoreto de sódio/monofluorfosfato de sódio. Em pH 6,8, ao se comparar a proteção dos quatro dentifricios teste (DI, DII, DIII e DIV) com o dentifricio controle CI (TAB. 3), os resultados obtidos revelaram diferenças estatisticamente significativas apenas entre o dentifrício teste DI e o dentifrício controle CI. Possivelmente, esta diferença deveu-se ao fato de este dentifrício teste ter sido o único a conter o fluoreto sob a forma de fluoreto de sódio. Muito embora o esmalte tratado pelo dentifrício fluoretado DIII, à semelhança do dentifrício fluoretado DI, tivesse liberado baixas taxas de cálcio, este dentifrício não apresentou diferença significativa em relação ao dentifrício controle $\mathrm{CI}$, conforme pode ser constatado na TAB. 3. Provavelmente, este fenômeno decorreu da presença da associação fluoreto de sódio/monofluorfosfato de sódio na composição do dentifrício teste DIII. Ainda em pH 6,8 (TAB. 3), apenas o dentifrício fluoretado DII não revelou diferença estatisticamente significativa em relação ao den- tifrício controle CII. Este resultado, possivelmente, decorreu da formulação do dentifrício fluoretado DII, caracterizado por conter apenas o monofluorfosfato de sódio na concentração de 1.200 ppmF $^{-}$. Muito embora o dentifrício teste DIV encerre apenas o monofluorfosfato de sódio na sua formulação, este produto revelou diferença estatisticamente significativa em relação ao dentifrício controle CII, provavelmente em decorrência da presença de 1.450 ppmF ${ }^{-}$, portanto uma concentração de fluoreto superior à contida no dentifrício teste DII.

$O$ fato de os dentifrícios fluoretados DI e DIII terem revelado diferenças estatisticamente significativas em relação ao dentifrício controle CII reafirma o grau de proteção do esmalte pelo fluoreto de sódio presente nesses produtos.

De acordo com a TAB. 1, com a queda do $\mathrm{pH}$ para 6,0, os dentifrícios fluoretados DI e DIII revelaram maior eficácia em face das mais baixas taxas do cálcio liberado, muito provavelmente, graças à presença do íon fluoreto sob a forma de fluoreto de sódio $(\mathrm{NaF})$. Embora os valores de cálcio decorrentes do esmalte tratado por estes dentifrícios não tivessem revelado diferenças estatisticamente significativas em $\mathrm{pH}$ $6,0(4,05 \pm 1,59$ e 8,94 $\pm 0,81$, respectivamente), possivelmente, a taxa mais baixa atribuída ao dentifrício fluoretado DI decorreu da eficácia do fluoreto de sódio $(\mathrm{NaF})$. Portanto, de acordo com a TAB. 3, tanto em relação ao dentifrício fluoretado DI quanto em relação ao dentifrício fluoretado DIII, os produtos fluoretados DII e DIV revelaram menor proteção do esmalte, expressas através das diferenças entre as taxas liberadas, consideradas estatisticamente significativas.

Ainda em pH 6,0 e de acordo com a TAB. 3, os dentifrícios fluoretados DI e DIII foram os que efetivamente protegeram o esmalte dentário humano. As baixas taxas do cálcio que foram liberadas do esmalte dentário influenciado por esses produtos revelaram diferenças estatisticamente significativas em relação aos dentifrícios controle CI e CII. Provavelmente, esse fenômeno resultou da presença do fluoreto de sódio no dentifrício teste DI e da associação 
fluoreto de sódio e monofluorfosfato de sódio no dentifrício teste DIII. Em pH 6,0, os dentifrícios teste DII e DIV revelaram a mesma influência propiciada pelos dentifrícios controle CI e CII. Possivelmente, o fato decorreu da presença do fluoreto, apenas sob a forma de monofluorfosfato de sódio em suas composições.

Ao se submeterem as amostras de esmalte ao $\mathrm{pH}$ 5,0 repetiu-se o mesmo fenômeno constatado em pH 6.0 (TAB. 3), ou seja: os dentifricios fluoretados DI e DIII, quando comparados entre si, libertaram taxas de cálcio distintas, muito embora a diferença de descalcificação não tenha sido estatisticamente significativa. Estes dentifrícios revelaram, entretanto, diferenças significativas em relação aos outros dois cremes dentais fluoretados. A repetição desse fenômeno parece reafirmar a maior eficácia do fluoreto de sódio $(\mathrm{NaF})$ sobre o monofluorfosfato de sódio (MFP).

Em relação aos dentifrícios controle CI e CII, mais uma vez os dentifrícios fluoretados DI e DIII revelaram proteger o esmalte dentário submetido ao pH 6,0, já que as diferenças entre os valores de cálcio foram estatisticamente significativas, em conformidade com os dados da TAB. 3. Os resultados obtidos em pH 6,0 demonstraram, ainda, que os dentifrícios teste DII e DIV exerceram a mesma influência demonstrada pelos dentifrícios controle CI e CII, uma vez que as diferenças entre as taxas de cálcio não foram consideradas significativas, do ponto de vista estatístico. Esse fenômeno parece reafirmar, uma vez mais, a eficácia do fluoreto de sódio $(\mathrm{NaF})$ e da associação entre o fluoreto de sódio e o monofluorfosfato de sódio ( $\mathrm{NaF}$ / MFP) sobre o monofluorfosfato de sódio (MFP) isoladamente.

A menor descalcificação constatada a partir do esmalte previamente tratado pelo dentifrício fluoretado DI, submetido ao $\mathrm{pH} 4,0$, confirma a maior eficácia do fluoreto de sódio $(\mathrm{NaF})$ sobre o monofluorfosfato de sódio (MFP), conforme está registrado nas TAB. 1 e 3. Em pH 4,0, portanto abaixo do nível crítico $(5,5)$, os dentifrícios que continham o fluoreto na forma de monofluor- fosfato de sódio (DII e DIV), da associação monofluorfosfato de sódio/fluoreto de sódio (DIII) ou que não continham fluoreto (CI e CII) não revelaram, in vitro, ação protetora sobre o esmalte dentário. Este resultado é evidenciado através das relevantes taxas do cálcio liberado do esmalte dentário influenciado por esses produtos, ao serem comparadas às taxas liberadas do esmalte dentário tratado pelo dentifrício fluoretado DI, cujas diferenças foram consideradas estatisticamente significativas.

Possivelmente, estes resultados revelados pelos dentifrícios decorreram da concentração do flúor nesses veículos, cujos valores se situam entre 1.100 e $1.500 \mathrm{ppmF}^{-}$, em média. Todavia a avaliação da influência protetora dos dentifricios fluoretados sobre o esmalte dentário humano em função dos valores decrescentes de $\mathrm{pH}$, ao colocar em evidência o dentifrício fluoretado DI, cujos valores de descalcificação obtidos em pH 6,8, 6,0, 5,0 e 4,0, além de terem sido os mais baixos, não revelaram diferenças estatisticamente significativas ao serem comparados entre si, reafirma a eficácia do fluoreto de sódio $(\mathrm{NaF})$ sobre as demais formas de fluoreto. Esta evidência é confirmada através do desempenho do dentifrício fluoretado DIII (associação fluoreto de sódio/monofluorfosfato de sódio), cuja descalcificação em pH 6,0 não acusa diferença estatisticamente significativa, em relação à descalcificação em pH 6,8. Esta diferença passa a ocorrer a partir do momento em que o esmalte dentário humano, previamente tratado pelo dentifrício fluoretado DIII, é submetido à ação do $\mathrm{pH}$ abaixo dos niveis criticos, ou seja: 5,0 e 4,0. A melhor proteção assegurada pelo fluoreto de sódio em relação ao monofluorfosfato de sódio é confirmada, também, em função da influência exercida pelos dentifrícios fluoretados DII e DIV nos quatro valores de $\mathrm{pH}$, uma vez que as taxas de cálcio obtidas em pH 6,0, 5,0 e 4,0 revelaram diferenças estatisticamente significativas em relação aos valores obtidos em $\mathrm{pH}$ 6,8, indicando, portanto, uma baixa proteção do esmalte dentário, in vitro, por esses dentifrícios fluoretados que continham apenas o monofluorfosfato de sódio (MFP). 
Estudos clinicos realizados anteriormente por De Paola et al. (1993) registram a eficácia dos dentifrícios fluoretados à base de fluoreto de sódio e de monofluorfosfato de sódio. Ao avaliar os efeitos de três dentifrícios fluoretados, após dois e três anos de uso por 2.222 crianças americanas, estes autores constataram a eficácia anticárie dos três produtos contendo $0,76 \%$ de monofluorfosfato de sódio, $0,24 \%$ e $0,243 \%$ de fluoreto de sódio, respectivamente.

A constatação, do presente estudo, da maior eficácia do dentifrício com fluoreto de sódio $(\mathrm{NaF})$ na proteção do esmalte dentário humano sobre os demais dentifrícios que contêm o monofluorfosfato de sódio (MFP), deveu-se, possivelmente, à ocorrência do íon fluoreto livre e totalmente aproveitável sob a forma de fluoreto de sódio $(\mathrm{NaF})$. Os resultados revelados pelo presente trabalho, realizado in vitro, estão de acordo com as conclusões de Bowen e Tabak (1995), ao relatar a superioridade dos dentifrícios contendo fluoreto de sódio em relação aos dentifrícios que contêm o monofluorfosfato de sódio. Segundo este autor, os dados in vitro e in situ, os estudos com roedores, os estudos farmacocinéticos em humanos e os ensaios clínicos controlados confirmam que os dentifrícios adequadamente formulados, contendo fluoreto de sódio, são mais efetivos do que os dentifrícios com monofluorfosfato de sódio na mesma concentração. Esta diferença é considerada clinicamente relevante.

Os resultados deste trabalho, ao comprovarem, in vitro, a maior eficácia do flúor sob a forma de fluoreto de sódio $(\mathrm{NaF})$ em relação ao monofluorfosfato de sódio (MFP), estão de acordo, também, com os resultados de pesquisas realizadas por Bowen e Tabak (1995), Marks et al. (1994) e Stephen et al. (1994).

De acordo com Bowen e Tabak (1995), o monofluorfosfato de sódio não possui esta biodisponibilidade. $\mathrm{Na}$ ausência das fosfatases, num sistema in vitro, o monofluorfosfato de sódio liberta o íon fluoreto muito lentamente, não revelando, portanto, uma efetividade comparável à demonstrada pelo fluoreto de sódio. In vivo, os níveis de fluoreto na placa e na sali- va, após o uso de dentifrícios contendo fluoreto de sódio em diferentes concentrações, confirmam a consistência da associação entre estes níveis e os efeitos clínicos. $O$ fato de o monofluorfosfato de sódio ser hidrolisado lentamente sobre as superfícies dentárias, na dependência da atividade catalítica das fosfatases, justifica a baixa concentração de fluoreto na placa, em relação à concentração detectada na placa de individuos que utilizam o fluoreto de sódio.

Estas constatações estão de acordo com as observações realizadas por Stephen et al. (1994), ao estudarem os efeitos de dentifrícios contendo fluoreto de sódio, monofluorfosfato de sódio e a associação fluoreto de sódio/trimetafosfato, utilizados durante três anos por adolescentes escoceses, sobre o incremento de cárie. Estes autores concluíram que o aumento médio do CPOS, após três anos, em indivíduos que usavam dentifrícios contendo apenas o fluoreto de sódio, foi $6,4 \%$ mais baixo em relação aos que usavam dentifrício contendo monofluorfosfato. Concluíram, também, que a diferença entre os indivíduos tratados pelo produto contendo a associação fluoreto de sódio/trimetafosfato de sódio e aqueles tratados com o monofluorfosfato de sódio foi da ordem de $8,1 \%$. O aumento médio do CPOS nos individuos que utilizaram o dentifrício contendo a associação fluoreto de sódio/ trimetafosfato de sódio foi $2 \%$ mais baixo do que o determinado para os individuos tratados com o dentifrício que possuía apenas o fluoreto de sódio, assim como a diferença entre o grupo tratado pela supracitada associação e aqueles tratados pelo monofluorfosfato foi da ordem de 2,3\%. Estes achados levaram os autores a afirmar que o fluoreto de sódio tem comprovada superioridade sobre o monofluorfosfato, quando incorporado aos dentifrícios bem formulados e com base de sílica.

Os resultados do presente estudo estão de acordo com os dados registrados por Stephen et al. (1994), uma vez que o dentifrício teste DI, tendo sido o produto fluoretado que revelou maior proteção do esmalte dentário humano submetido aos quatro valores de $\mathrm{pH}(6,8$, $6,0,5,0$ e 4,0) é caracterizado por possuir o 
ín fluoreto apenas na forma de fluoreto de sódio (1.100 $\left.\mathrm{ppmF}^{-}\right)$, tendo a sílica como base.

O fato de o dentifrício fluoretado DIII ter sido o único produto a revelar a eficácia protetora mais próxima à revelada pelo dentifrício fluoretado DI está de acordo com os achados de Stephen et al. (1994), uma vez que, além do monofluorfosfato de sódio, o dentifrício DIII possui, também, o fluoreto de sódio.

Outrossim, estudos clínicos realizados por Marks et al. (1994), comparando a eficácia de dentifrícios contendo fluoreto de sódio e monofluorfosfato de sódio, confirmam as conclusões de Stephen et al. (1994) e de Bowen e Tabak (1995). O comprovado poder de proteção do fluoreto sobre o esmalte dentário humano, fundamentado no modelo experimental in vitro adotado no presente estudo, está de acordo com as conclusões registradas por estes autores.

Ao avaliar os resultados do uso de dentifrícios contendo esses produtos, durante três anos, por crianças residentes na Flórida, Marks et al. (1994) detectaram que, no grupo tratado pelo fluoreto de sódio $\left(2.000 \mathrm{ppmF}^{-}\right)$, houve um aumento significativamente menor do CPOS em relação ao grupo tratado pelo monofluorfosfato de sódio (2.000 $\mathrm{ppmF}^{-}$). Através deste estudo, os autores concluiram uma superioridade significativa $(\mathrm{p}<0,005)$ do fluoreto de sódio sobre o monofluorfosfato de sódio, assim como uma clara relação dose-resposta entre a eficácia anticárie e o nível de fluoreto dos dentifrícios contendo em suas formulações o monofluorfosfato de sódio nas concentrações de $1.000,1.500,2.000$ e 2.500 $\mathrm{ppmF}^{-}$. Estes autores concluíram que a eficácia clínica do dentifrício teste contendo 2.000 $\mathrm{ppmF}^{-}$, sob a forma de fluoreto de sódio, parece ser semelhante à eficácia revelada pelos dentifrícios teste contendo monofluorfosfato de sódio em concentração de $2.500 \mathrm{ppmF}^{-}$.

A menor eficácia dos dentifrícios fluoretados DII e DIV deveu-se, provavelmente, à presença, nesses produtos, do fluoreto apenas sob a forma de monofluorfosfato de sódio. Muito embora esses dentifrícios encer- rem, no conteúdo de suas formulações, concentrações de flúor (1.500 $\mathrm{ppmF}^{-}$e 1.450 ppmF-, respectivamente) superiores à concentração do flúor presente no dentifrício teste DIII $\left(1.200 \mathrm{ppmF}^{-}\right)$, a maior eficácia atribuida a este último dentifrício decorreu, possivelmente, da presença do fluoreto de sódio associado ao monofluorfosfato de sódio.

É extremamente importante a continuidade de novas experimentações visando subsidiar, cada vez mais, os dados da literatura cientifica que tratam da eficácia protetora do fluoreto de sódio, do monofluorfosfato de sódio e da associação entre estes dois agentes, em face da importância de se obterem respostas preventivas e terapêuticas mais eficazes com a utilização de baixas concentrações de fluoreto.

\section{Conclusões}

A partir dos resultados aqui apresentados, pode-se concluir que:

1) o dentifrício contendo a associação de fluoreto de sódio e monofluorfosfato de sódio $\left(1.500 \mathrm{ppmF}^{-}\right)$parece ter induzido proteção do esmalte dentário humano, em pH 6,8 e 6,0. Contudo, esta proteção foi relevante em pH 5,0, ao se comparar a ação desse dentifrício com dois produtos não fluoretados;

2) o dentifrício contendo monofluorfosfato de sódio $\left(1.450 \mathrm{ppmF}^{-}\right)$possivelmente só induziu proteção do esmalte dentário humano em $\mathrm{pH}$ 6,8, enquanto o dentifrício contendo monofluorfosfato de sódio $\left(1.200 \mathrm{ppmF}^{-}\right)$não induziu, provavelmente, proteção do esmalte dentário humano na faixa de $\mathrm{pH}$ 6,8 a 4,0;

3) o dentifrício contendo fluoreto de sódio (1.100 $\mathrm{ppmF}^{-}$) parece ter induzido proteção do esmalte dentário humano frente aos valores de $\mathrm{pH} 6,8,6,0,5,0$ e 4,0 com a mesma intensidade. Este mesmo grau de proteção repetiu-se ao se comparar a ação deste dentifrício com dois dentifrícios não fluoretados; 
4) os dentifrícios que possuem fluoreto de sódio induzem, possivelmente, proteção ao esmalte dentário humano com mais intensidade, uma vez comparados aos que possuem, em suas formulações, o monofluorfasfato de sódio ou a associação entre o fluoreto de sódio e o mono- fluorfosfato de sódio. Essa proteção mais eficaz do fluoreto de sódio provavelmente ocorre até mesmo em concentrações mais baixas de flúor, em relação às demais formas, mesmo que os valores de $\mathrm{pH}$ do meio venham a ocorrer abaixo dos níveis considerados críticos.

\begin{abstract}
The possibility of interferring in the dental enamel solubility, reducing significatively the mineral loss, and the possibility of reverting the progress of initial carious lesions turn the fluoride an absolutely necessary element for the oral health. As preventive alternative against caries, the fluoride constitutes of an extremely efficient option for oral public healthcare at low cost. To achieve the purpose of supporting the literature concerning the efficacy of sodium fluoride (NaF), SMFP and of the association between NaF and SMFP present in dentifrices and mouthrinses, the present study aimed to determine in vitro the protection level of the human dental enamel provided by these fluoride forms in presence of $\mathrm{pH}$ drop. The rates of calcium removed from the enamel, which had been previously treated, demonstrated these drugs' protecting action. The experimental results prove that the $\mathrm{NaF}$ contained in the test-dentifrice revealed to provide greater enamel protection, despite the lower concentration of this product when compared to the SMFP and to the NaF/SMFP association. These conclusions suggest the relevance of continuing those investigations, due to the importance of obtaining efficient answers by using low concentration of the most effective fluoride form as well as the importance of submitting the different products, frequently introduced to the market, to a quality control process.
\end{abstract}

\title{
Key words
}

Fluoride, dentifrice, SMFP, dental enamel, remineralization

\section{Referências}

ARAÚJO, R. P. C. Odonto-rural: uma proposta alternativa de saúde bucal. Cadernos do IFUFBA, v. 7, n. 1-2, p. 197209, 1995.

BARTLETT, D. M.; SMITH, B. G.; WILSON, R. F. Comparison of the effect of fluoride and non-fluoride toothpaste on tooth wear in vitro and the influence of enamel fluoride concentration and hardness of enamel. Br. Dent. J., v. 176, n. 9, p. 346-348, 1994.

BJARNASON, S. et al. Caries experience in icelandic 12year-old urban children between 1984 and 1991 . Community Dent. Oral Epidemiol., v. 21, 1. 4, p. 195-197, 1993.

BOWEN, W. H.; TABAK, L. A. Cariologia para a década de 90. Tradução de Sérgio Wayne e Ruy Oppermman. São Paulo: Santos, 1995. 462p.

CHADWICK, S. M.; GORDON, P. H. An investigation to estimate the fluoride uptake adjacent to fluoride releasing bonding agent. Br. J. Orthod., v. 22, n. 2, p. 113-122, 1995.

CHRISTOFFERSEN, J. et al. Formation of phosphate containing calcium fluoride at the expense of enamel, hydroxyapatite and fluorapatite. Caries Res., v. 29, n. 3, p. 223-230, 1995.

CRUZ, R.; RÖLlA, G.; ÖGAARD, B. Alkali soluble fluoride disposition on human enamel exposed to monofluorophosphate containing tootpastes in vitro. Acta Odontol. Scand., v. 52, n. 2, p. 72-76, 1994a.
CRUZ, R.; RÖLLA, G. A scanning electron microscope investigation of calcium fluoride like material deposited during topical fluoride exposure on sound human enamel in vitro. Braz. J. Med. Biol. Res., v. 27, n. 10, p. 2371-2377, $1994 b$.

CURY, J. A. Uso do flúor. In: BARATIERI, L. N. Dentística: procedimentos preventivos e restauradores. 2. ed. São Paulo: Santos, 1989. p. 43-67. il.

CUTRESS, T. W. Effects of fluoride-supplemented suciose on experimental dental caries and dental plaque $\mathrm{pH}$. Adv. Dent. Res., v. 9, n. 1, p. 14-20, 1995.

DE PAOLA, P. F. Clinical studies on MFP/calcium containing abrasive. Int. Dent. J., v. 43, n. 1, suppl. 1, p. 89 96, 1993.

ELLWOOD, R. P.; BLINKHORN, A. S.; DAVIES, R. M. Fluoride: how to maximize the benefits and minimize the risks. Dental Update, v. 25, p. 365- 372, 1998.

FISCHER, C.; LUSSI, A.; HOTZ, P. The cariostatic mechanism of action of fluorides: a review. Schweiz Monatsschr Zahnmed, v. 105, n. 3, p. 313-317, 1995.

GAFFAR, A.; BLAKE-HASKINS, J.; MELLBERG, J. In vivo studies with a dicalcium phosphate dihydrate / MFP systems for caries prevention. Int. Dent. J., v. 43, n. 1, suppl. 1, p. 81-88, 1993.

HAWLEY, G. M. A 30 month study investigating the effect of adding triclosan / copolymer to a fluoride dentifrice. Caries Res., v. 29, n. 3, p. 163-167, 1995. 
IMFELD, T. et al. Fluoride gels-local application with a tray or a toothbrush. Schweiz Monatsschr Zahumed, v. 103, n. 11, p. 1401-1406, 1993.

JOYSTON-BECHAL, S. et al. Caries incidence mutans streptococci and lactobacilli in irradiated patients during a 12 month preventive programme using chlorhexidrine and fluoride. Caries Res., v. 26, p. 384-396, 1992.

JOYSTON-BECHAL, S.; KIDD, E. A. M. Update on the appropriate user of fluoride. Dental Update, v. 21, p. 366371, 1994.

KAMBARA, M.; NORDE, W. Influence of fluoride applications on some physicochemical surface properties of synthetic hydroxyapatite and human dental enamel and its consequences for protein adsorption. Caries Res., v. 29, n. 3, p. 210-217, 1995

KINGMAN, A. Methods of projecting long term relative efficacy of products exhibiting small short-term efficacy. Caries Res., v. 27, n. 4, p. 322-327, 1993.

$\mathrm{KOCH}$, G. et al. Effect of 250 and 1000 ppm fluoride dentifrice on caries: a three-year clinical study. Swed. Dent. J., n. 6, p. 233-238, 1982.

KRASSE, B. Risco de cáries: um guia prático para avaliação e controle. São Paulo: Quintessence, 1988. 113p. il.

KUROIWA, M.; KODAKA, T.; KUROIWA, M. Microstructural changes of human enamel surfaces by brushing with and without dentifrice containing abrasive. Caries Res., v. 27, n. 1, p. 1-8, 1993.

LEVY, S. M. A review of fluoride intake from fluoride dentifrice. ASDC J. Dent. Child., v. 60, n. 2, p. 115-124, 1993.

LEVY,S. M.; MAURICE, T. J.; JAKOBSEN, J. R. Dentifrice use among preschool children. J. Am. Dent. Assoc., v. 124, n. 9, p. 57-60, 1993.

LOESCHE, W. J. Cárie dental: uma infecção tratável. Rio de Janeiro: Cultura Médica, 1993. 349p. il.

LUOMA, H. et al. Enamel dissolution in relation to fluoride concentrations in the fluid of dental plaque-like layers of pre-cultured streptococus sobrinus. Arch. Oral Biol., v. 39, n. 3, p. 177-184, 1994.

MARKS, R. G. et al. Results from a three-years caries clinical trial comparing $\mathrm{NaF}$ and SMFP fluoride formulations. Int. Dent. J., v. 44, n. 3, suppl. 1, p. 275-285, 1994.

NEWBRUN, E. Agentes anticárie e antiplaca: antigengivite. In: NEIDLE, E. A.; YAGIELA, J. A. Farmacologia terapêutica para dentistas. Rio de Janeiro: Guanabara Koogan, 1991. p. 483-494. 608 p. il.
PALENSTEIN, W. H. et al. Two types of intraoral distribution of fluorotic enamel. Community Dent. Oral Epidemiol., v. 25, n. 3, p. 251-255, 1997.

PETERSSON, L. G. et al. The effect of low fluoride containing toothpaste on the development of dental caries and microbial composition using a caries generating model device in viwo. Swed. Dent. J., v. 19, n. 3, p. 83-94, 1995.

PINHEIRO, C. E. Efeito da associação cloreto de cetilpiridinio-fluoreto de sódio na fermentação e na sintese de polissacarideos extracelulares insolúveis da placa dentária in vitro. Rev. Bras. Odontol., v. 48, p. 18-20, 1991.

RIORDAN, P. J. Fluoride supplements in caries prevention: a literature review and proposal for a new dosage schedule. J. Public Health Dent., v. 53, n. 3, p. 174-189, 1993.

SERRA, M. C.; SARTINI FILHO, R.; CURY, J. A. Incorporação e retenção de flúor em esmalte e dentina após aplicação tópica de flúor fosfato acidulado. Rev. Bras. de Odonto., v. 46, n. 1, p. 18-23, 1989.

SHELLIS, R. P.; DUCKWORTH, R. M. Studies on the cariostatic mechanisms of fluoride. Int. Dent. J., v. 44, n. 3 , suppl. 1, p. 263-273, 1994.

STEPHEN, K. W. et al. The effect of NaF and SMFP toothpastes on three-years caries increments in adolescents. Int. Dent. J., v. 44, n. 3, suppl. 1, p. 287-295, 1994.

TEN CATE, J. M. The caries preventive effect of fluoride dentifrice containing triclosan and zinc citrate, a compilation of in vitro and in situ studies. Int. Dent. J., v. 43, n. 4, suppl. 1, p. 407-415, 1993.

TEN CATE, J. M.; MARSH, P. D. Procedures for establishing efficacy of antimicrobial agents for chemotherapeutic caries prevention. J. Dent. Res., v. 73, n. 3, p. 695-703, 1994.

THYLSTRUP, A.; FEJERSKOV, O. Cariologia clínica. Tradução de Sérgio Weyne. 2. ed. São Paulo: Santos, 1995. 421 p.

WALKER, C. The effects of a $0,3 \%$ triclosan containing dentifrice on the microbial composition of supragingival plaque. J. Clin. Periodontol., v. 21, n. 5, p. 334-341, 1994.

WILTSHIRE, W. A.; Van RENSBURG, S. D. J. Fluoride release from four visible light-cured orthodontic adhesive resins. Am. J. Orthod. Dentofacial Or thop., v. 108, n. 3, p. 278-283, 1995.

YATES, R. et al. A 6-month home usage trial of a $1 \%$ chlorhexidrine toothpaste (1): effects on plaque, gingivitis, calculus and toothstaining. J. Clin. Periodontol., v. 20, p. 130-138, 1993. 